\title{
A 2-AXIS QUASI-PASSIVE PLATFORM FOR NANOSCALE PHOTONIC ASSEMBLY
}

\author{
Biao Li, Marco Pietrusky, Andre Sharon \\ Fraunhofer USA Center for Manufacturing Innovation \\ 15 St. Mary’s St., Brookline, MA 02446, USA
}

\begin{abstract}
As the need for communication bandwidth continues to increase and devices move from multi-mode to single-mode technology, optical alignment requirements are becoming more stringent, approaching the nanometer level. We previously reported a 1-axis quasi-passive platform with nanoscale alignment tolerance [1]. This paper presents our research on a 2-axis positioning platform capable of four degrees-of-freedom in-plane motion. Particularly, a modified design has been adapted for rapid device production. In addition, a focused ion beam (FIB) technique has been utilized for in-situ stress element trimming and nanoscale motion characterization. Furthermore, an automated program has been implemented for light coupling experiment. Finally, the reliability of the 2-axis platforms has been evaluated.
\end{abstract}

\section{INTRODUCTION}

Currently, active alignment remains as the only viable means for low-loss optical coupling with the required alignment tolerance $(<100 \mathrm{~nm})$ [2]. The problems associated with this costly process are well known to those in photonic packaging including complex fiber handling and post-alignment shift. Silicon optical bench (SiOB) technology emerged as a means to passively align and integrate optical components on an optical breadboard [3]. However, progress in the development of SiOB technology has been slow due to the difficulties in aligning the optical path to nanometer-scale tolerance often required. Using purely passive locating features, one can position components on a micro-optical bench to desired locations relative to one another within a tolerance of at best $1 \mu \mathrm{m}$. This is an order of magnitude worse than what is needed for efficiency coupling between devices and/or fibers.

Inspired by resistor trimming in microelectronics, we developed a quasi-passive integration technique capable of nanometer-scale positioning tolerances. The concept capitalizes on inherent residual tensile stresses in MEMs thin films, and comprises a platform suspended by stressed flexure elements on either side. These thin film flexures, made of highly stressed materials such as silicon nitride, can support in-plane tensile stresses up to $\sim 1 \mathrm{GPa}$. By selectively trimming stress elements, the equilibrium position of the platform can be biased to one side or another, enabling high resolution relative motion between the suspended platform and the base. The movement of the platform can be directed toward the light path using techniques of active alignment but without necessitating any micro-handling of the components or fibers, and without necessitating subsequent immobilization. The scale of this adjustment is well suited to the alignment task, yielding nanometer-scale resolution with sufficient range for typical applications.

As a proof of concept, we previously reported a 1-axis quasipassive platform capable of sub-micron alignment optimization [1]. This paper presents our research on a 2-axis positioning platform with emphasis on stress element design, nanoscale motion characterization, laser trimming automation, and robustness enhancement.

\section{FINITE ELEMENT MODELING}

Figure 1 shows the finite element modeling (FEM) of the platform displacement in response to trimming the stress elements. The 2-axis platform enables four in-plane degrees-of-freedom two in translation, and two in angular rotation. Translation can be achieved by cutting pairs of stress elements symmetrically; while angular motion can be achieved by asymmetrically trimming the stress element. The effect of cutting a stress element on one side of the platform is identical to that of pulling the corresponding one on the other side. FEM also indicates that more stress elements facilitate higher resolution motion; while fewer stress elements facilitate larger displacement. Nanometer-scale platform movement is reachable with a large number of short stress elements.

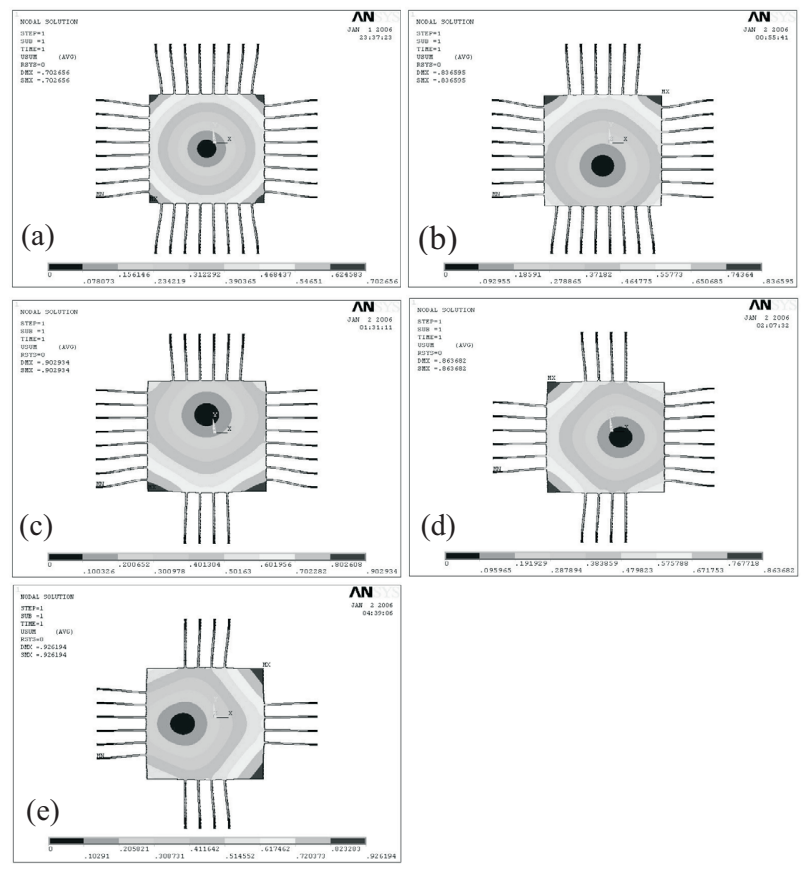

Figure 1: Finite element simulation of the nanoscale platform motion in response to trimming the stress element. (a) Initial position; (b) downward motion; (c) upward motion; (d) rightward motion; and (e) leftward motion.

\section{EXPERIMENTAL DETAILS}

I. Rapid production of 2 -axis platform. We have manufactured 2-axis devices using standard microfabrication technology. The process started with polished [100] orientation silicon ( $\mathrm{Si}$ ) wafers. Prior to low-pressure chemical vapor deposition (LPCVD), standard wafer cleaning was applied. A 200 $\mathrm{nm}$ thick stoichiometric $\mathrm{Si}_{3} \mathrm{~N}_{4}$ thin film was grown from $30 \mathrm{sccm}$ dichlorosilane (DCS) and $90 \mathrm{sccm}$ ammonia at $825{ }^{\circ} \mathrm{C}$ under a pressure of $300 \mathrm{mTorr}$. This resulted in an inherent tensile stress of approximately $1 \mathrm{GPa}$ in the film. The platform and stress element 
patterns were formed after selectively reactive ion etching the frontside $\mathrm{Si}_{3} \mathrm{~N}_{4}$ film. Consequently, etching windows were created after selectively etching backside $\mathrm{Si}_{3} \mathrm{~N}_{4}$ film. Finally, the patterned wafers were put into a potassium hydroxide $(\mathrm{KOH})$ bath to release the $\mathrm{Si}_{3} \mathrm{~N}_{4}$ from the surrounding $\mathrm{Si}$ structure.
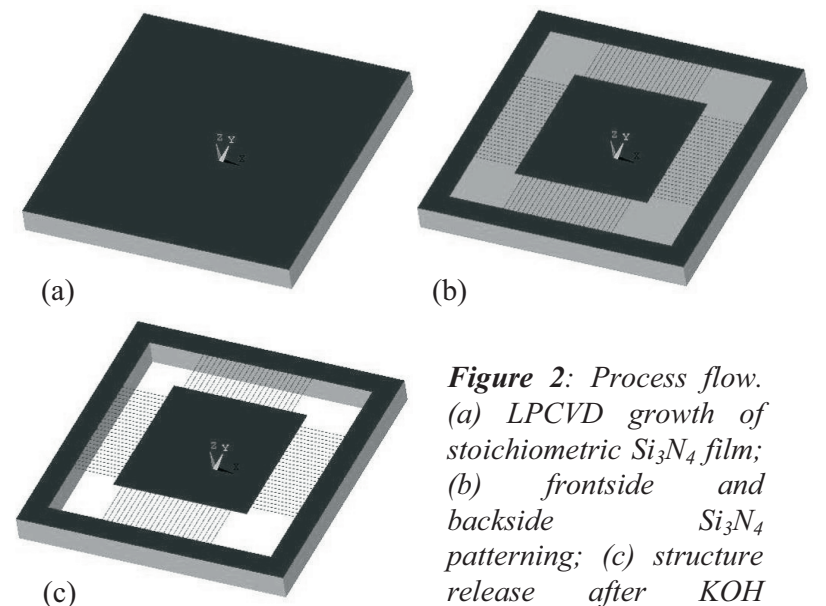

Figure 2: Process flow. (a) LPCVD growth of stoichiometric $\mathrm{Si}_{3} \mathrm{~N}_{4}$ film; (b) frontside and backside $\quad \mathrm{Si}_{3} \mathrm{~N}_{4}$ patterning; (c) structure release after $\mathrm{KOH}$ etching.

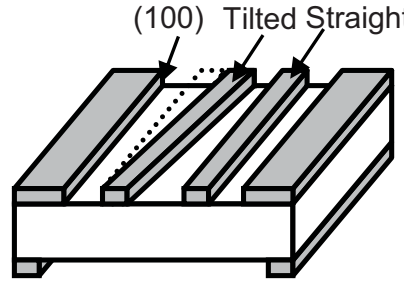

(a)

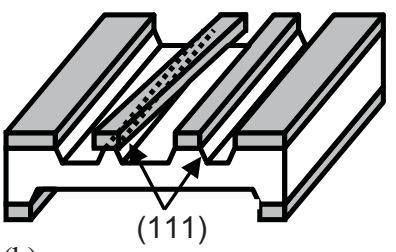

(b)

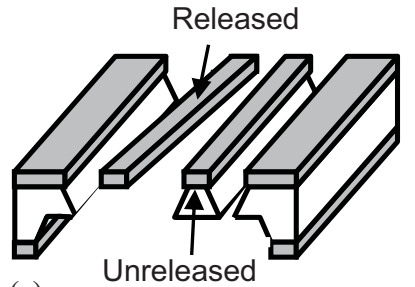

(c)

Unreleased
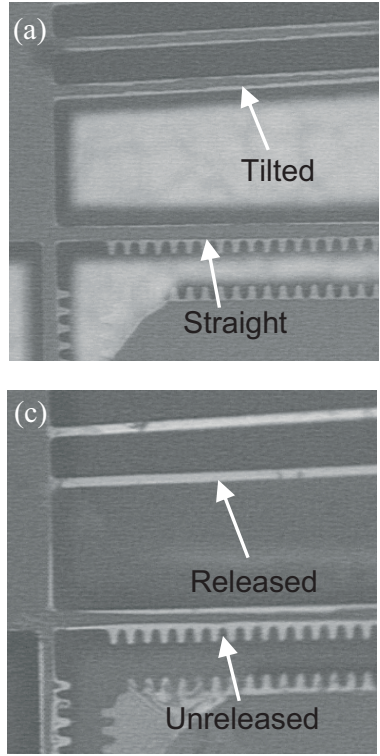

Figure 4: The undercut etching of the stress elements after etching for (a) $35 \mathrm{~min}$, (b) 55 min and (c) 70 min in $\mathrm{KOH}$.
To activate the stressed-element, all $\mathrm{Si}$ underneath the $\mathrm{Si}_{3} \mathrm{~N}_{4}$ film must be completely removed. It is found that the release of $\mathrm{Si}_{3} \mathrm{~N}_{4}$ always took a great deal of time for a straight configuration where all stress elements were oriented along the (100) direction of the $\mathrm{Si}$ wafer (Figures $3 \& 4$ ). As such, the free-suspended structures were often destroyed during the long-term aggressive etching process. On the other hand, a tilted configuration where stress elements were misoriented from the $(100)$ orientation of $\mathrm{Si}$ wafers would be subjected to severe undercut etching. We exploited this characteristic for rapid production of a 2-axis platform. Figure 5 illustrates a micro-machined 2-axis platform. The tilted stress elements were completely released due to quick undercut etching.

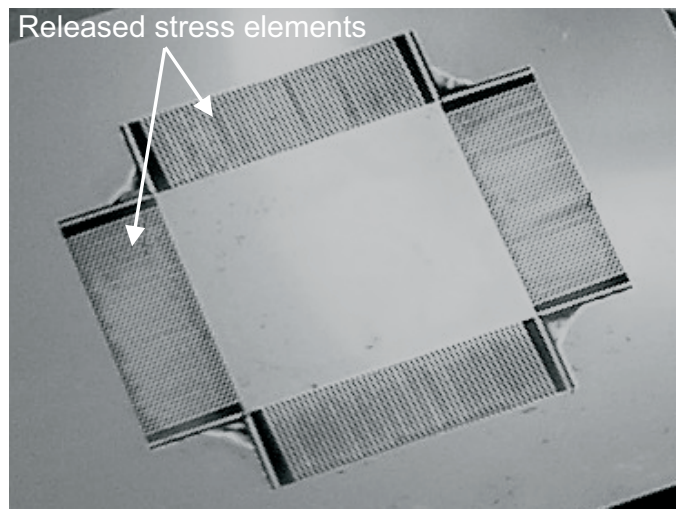

Figure 5: Optical photograph of a micro-machined 2-axis platform with completely released stress elements.

II. Focused ion beam trimming and characterization. A focused ion beam (FIB) technique [4] was utilized for in-situ stress element trimming and nanoscale motion characterization. The experiment was conducted in an FEI FIB 200 system. As shown in Figure 6a, the 2-axis platform was first epoxy glued on a polished $\mathrm{Si}$ piece before transported into vacuum chamber. The Si piece provided not only a conductive substrate for effective charge neutralization but also a clear background for nanoscale imaging. A focused ion beam ejected from a liquid Gallium $(\mathrm{Ga})$ ion source, with a spot size of less than $10 \mathrm{~nm}$, is scanned across the sample with $30 \mathrm{kV}$ kinetic energy. At a beam current of $70 \mathrm{pA}$ and with a magnification of 2000, individual stress element could be selectively etched within one minute. Figure $6 \mathrm{~b}$ shows a FIB trimmed stress element.

To evaluate the platform motion with respect to stress element trimming, we created several trenches on the underlying Si piece using FIB milling. Prior to FIB processing, patterning geometry of $10 \mu \mathrm{m} \times 1 \mu \mathrm{m} \times 1 \mu \mathrm{m}$ was defined at the location of $10 \mu \mathrm{m}$ away from the platform edge. Using a beam current of 70 $\mathrm{pA}$ and a magnification of 6500 , trenches were generated on Si. Figure $6 \mathrm{c}$ illustrates a FIB milled trench that is close to the upper midpoint of the platform. Images were recorded using secondary electron contrast with a magnification of 6500 (corresponding to a pixel spacing of $11 \mathrm{~nm}$ ).

Figure 6d illustrates the platform movement after symmetrically cutting four stress elements on the upper side. With more stress elements pulling below the platform, the FEM predicted a net movement of $195 \mathrm{~nm}$ downwards. To determine the true position shift of the platform after trimming the stress elements, we attempted to align the trench at different images to the same horizontal level (guideline 1). The positions of platform were then identified by guidelines 2 and 3. The FEI 200 system 
provides the ability of fine-tuning the magnification $( \pm 1 \times)$. Thus, imaging distortion during different scans is neglected. Using a 10 $\mu \mathrm{m}$ scale bar as a reference, the distance between guidelines 2 and 3 was measured as $-206 \mathrm{~nm}$. Since the measurement error for each guideline is one pixel size, $\pm 11 \mathrm{~nm}$, the total error of the measurement is $\pm 33 \mathrm{~nm}$. This indicated that the platform behaved as modeled, resulting in a net movement of approximately $206 \mathrm{~nm}$ $( \pm 33 \mathrm{~nm})$ downwards.
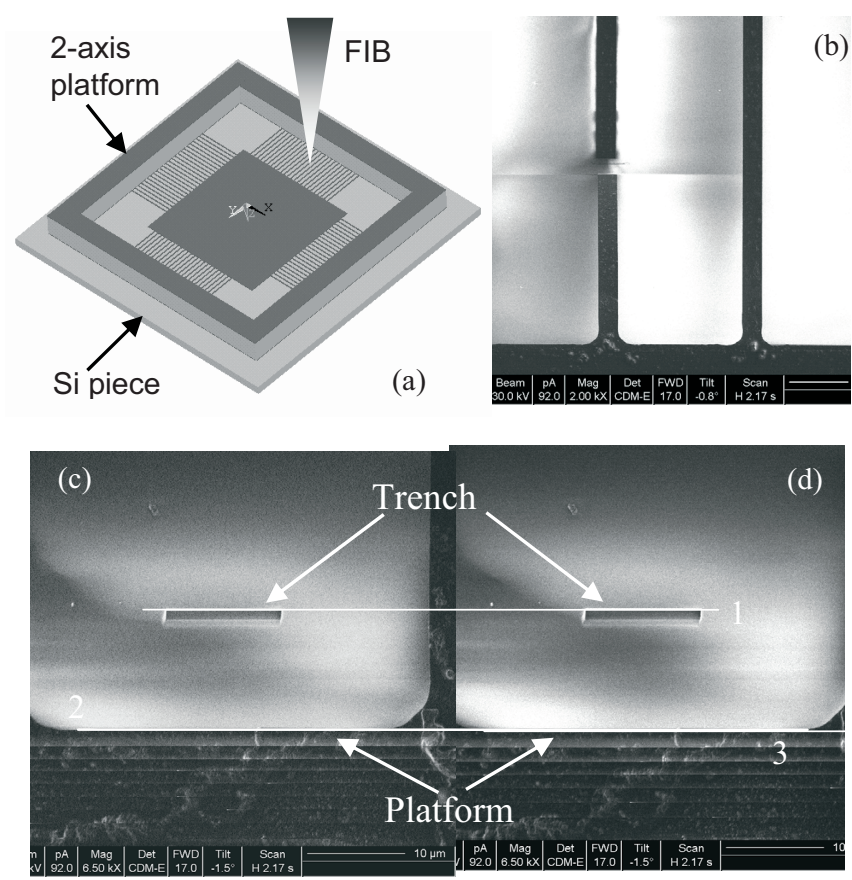

Figure 6: Nanoscale motion characterization. (a) A 2-axis platform is placed on a polished Si piece for FIB trimming and imaging; (b) A stress element is FIB cut; (c) A maker is FIB machined on the underneath Si piece. (d) Downward platform motion after FIB trimming four stress elements.

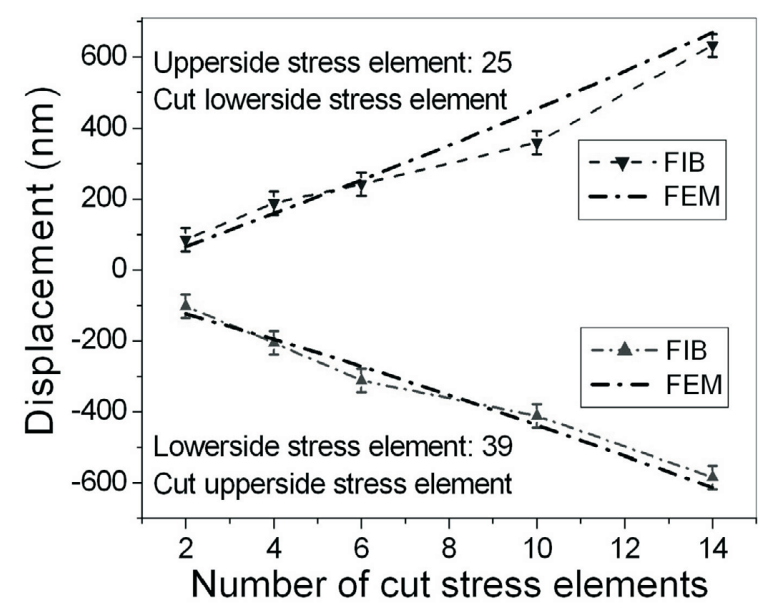

Figure 7: Platform motion with respect to trimming stress elements. The measured data (error bar: $\pm 33 \mathrm{~nm}$ ) agrees well with the finite element simulation results.

Figure 7 exhibits the platform motion as the result of removing the stress elements at the upper- and lower-side of the platform, respectively. The dots-dashed-lines represent the experimental data derived from FIB measurement, while the solid lines refer to the FEM results. The measured displacement agrees well with the finite element modeling, validating this as a predictable device for nanoscale motion management. In addition, the translation is almost linear and reversible with respect to the number of cut stress elements. With 39 stress elements on the lower side, trimming one stress element on the upper side of the platform generates approximately $38 \mathrm{~nm}$ downward motion. The upward motion is realized by cutting the stress elements on the lower side of the platform. The average upward motion step, 42 $\mathrm{nm}$, is slightly larger than the downward motion step because only 25 stress elements exists on the opposite side.

III. Automated fiber coupling experiment. We tested the two-axis device in a real functional application, namely the alignment of two single-mode optical fibers to one-another. In our prior work [1], a scanning laser system was employed for characterizing the 1-axis platform, in which SU-8 grooves were patterned for single-mode fiber attachment. Since a $\sim 4 \mu$ thick ProTEK protection layer was necessary to maintain SU-8 intact during $\mathrm{KOH}$ etching, the subsequent removal of this layer would always break the stress elements. In the current approach, we utilized two $\mathrm{x}-\mathrm{y}-\mathrm{z}-\theta$ stages to manipulate two single-mode fibers attaching respectively to the suspended platform and the stationary base of the 2-axis device. After pre-alignment, the fibers were epoxy glued for light coupling characterization (Figure 8a).
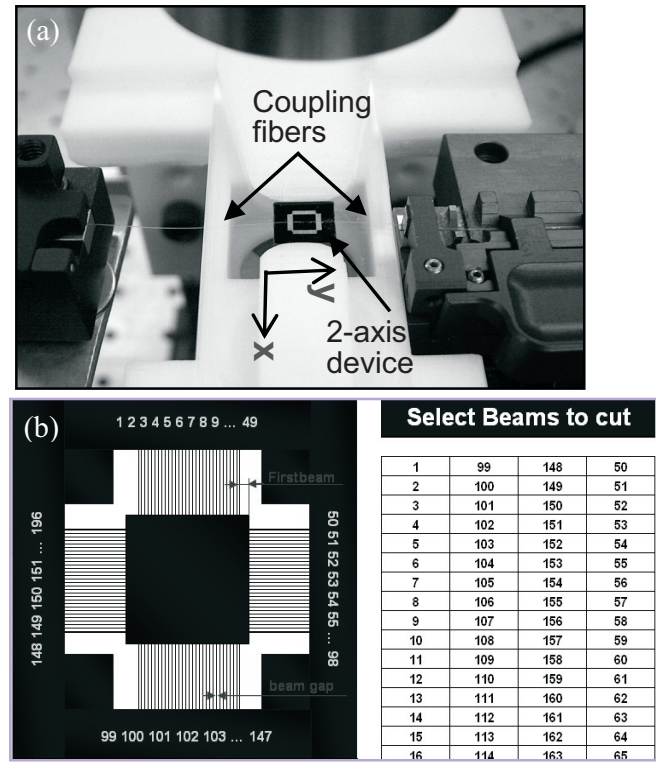

Figure 8: (a) Fiber coupling experiment. The opposite end of a single-mode fiber attached to a laser diode is mounted on the suspended platform, while the opposing end of a fiber attached to a power meter is mounted on the stationary base of the substrate. Selective laser trimming of the stress elements enables nanoscale motion between the two fibers. (b) Interface of laser trimming system.

We developed an automated program for laser trimming. As shown in Figure-8, the computer interface allows the selection of any stress element. A visual-basic program translates the code into ScanWare for the actual firing of laser pulse. This enables rapid, flexible, and straightforward command of platform position. In our experiment, the fibers were placed in parallel to the y-axis stress elements. Selective laser trimming of the stress elements enabled 2 -axis nanoscale motion between the two fibers. 
Figure 9 displays an alignment process resulting from selectively trimming the 2-axis stress elements using programmed laser pulsing. The experiment was performed by cutting the stress elements in $\mathrm{x}$-axis and $\mathrm{y}$-axis, respectively, which led to lateral and axial positioning of the aligned fiber. The resulting light passing from one fiber to the other was then measured. The relative position steps were not actually measured, but determined from finite element simulation. Obviously, the alignment of the two fibers can be optimized for maximum coupling. In addition, the coupling efficiency is more sensitive to the shift of lateral position (x-axis) than axial position (y-axis).

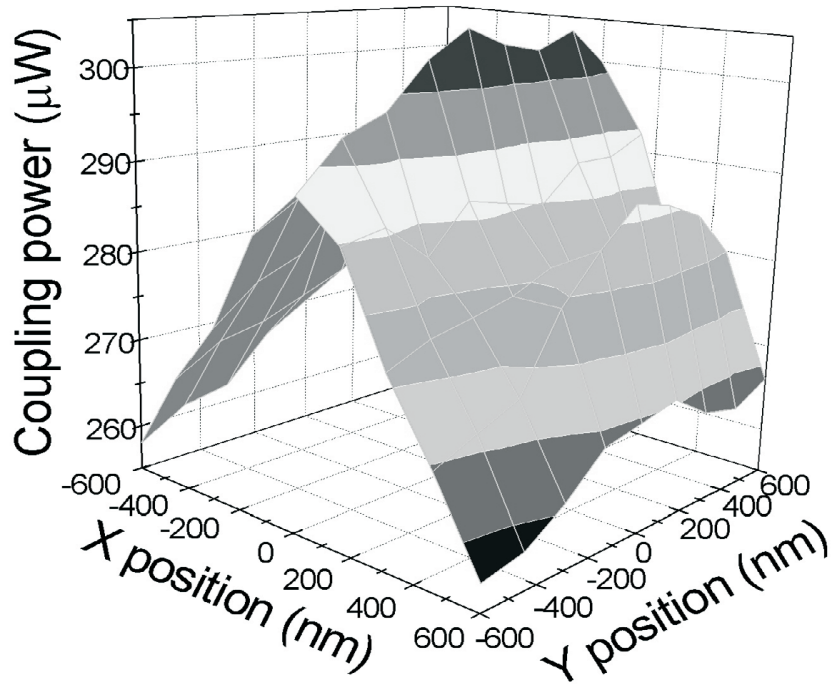

Figure 9: Fiber coupling improvement resulting from selectively trimming 2-axis stress elements. The coupling power is more sensitive to lateral ( $x$-displacement) than axial (y-displacement) changes.

IV. Reliability evaluation of the $\mathbf{2}$-axis platform. Finally, we performed finite element modeling to evaluate the reliability of the 2-axis platform based on Telcordia standards. To analyze if the device was robust enough against external unexpected shocks, we derived von Mises equivalent stresses with ANSYS modeling, and then solved for the acceleration level necessary to reach a given level of fracture strength $\left(\sim 7 \mathrm{GPa}\right.$ for $\mathrm{SiN}_{\mathrm{x}}$ [5]) for various stress element length and number. It is seen from Figure 10a that long stress elements require large shock levels to achieve the fracture stress. From Figure 11a it is also clear that a large number of stress elements require a high level of shock to achieve the fracture stress. The simulation predicts that the 2 -axis platform is able to survive under a $3000 \mathrm{~g}$ shock.

The thermal stability of the 2-axis platform was also analyzed. Shown in Figure 10b and Figure $11 \mathrm{~b}$ is the displacement of the platform center at two typical temperature extremes for thermal cycling, $-40{ }^{\circ} \mathrm{C}$ and $+80{ }^{\circ} \mathrm{C}$, respectively. The temperature variation modifies the positioning characteristics only approximately a few nanometers. The remarkable thermal stability of the 2-axis platform is attributed to the tensile constraint from the 2-axis stress elements.

We further calculated the resonant frequency of the 2-axis platform with different configurations. As shown in Figure 10c and Figure $11 \mathrm{c}$, the resonant frequency raises with the increasing number and the decreasing length of stress elements. The overall frequency is more than $10 \mathrm{KHz}$. This is far above typical vibration frequency ranges $(20 \mathrm{~Hz}$ to $2000 \mathrm{~Hz})$. All this validates the potential of the 2-axis platform for nanoscale photonic packaging.

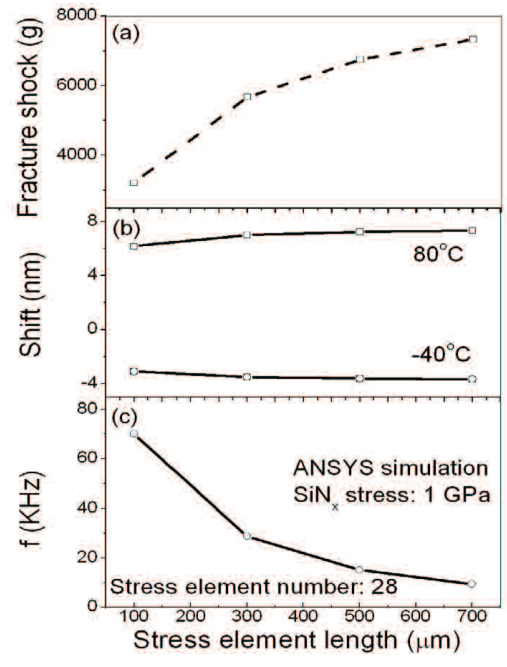

Figure 10: Dependence of (a) shock resistance, (b) thermal stability and (c) resonant frequency on stress element length.

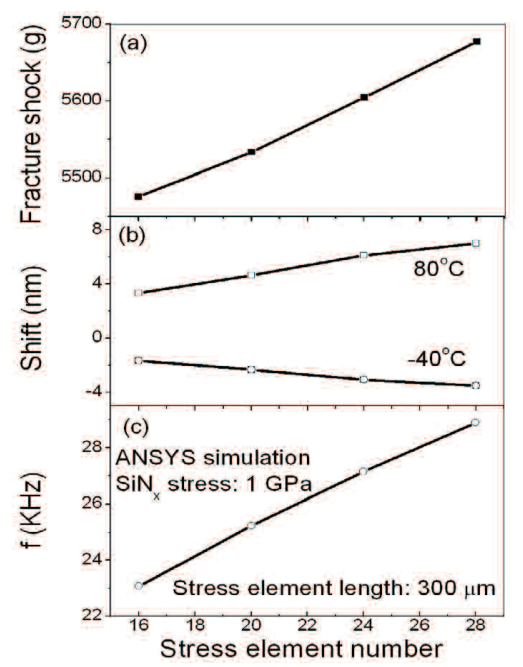

Figure 11: Dependence of (a) shock resistance, (b) thermal stability, and (c) resonant frequency on stress element number.

\section{REFERENCES}

[1] B. Li, J. Menger, T. Walsh, H. Wirz, A. Sharon, "Development of quasi-passive optical substrates for photonic packaging", Proceeding of the 18th IEEE International Conference on Micro Electro Mechanical Systems (MEMS '05), Miami Beach, FL, USA, 01/30-02/03, (2005), pp. 104-107

[2] A. Goding, "Active alignment is here to stay", Photonics Spectra, pp. 110-112 (2002)

[3] W. Shakespeare, R. Pearson, J. Grenestedt, P. Hutapea, V. Gupta, "MEMS integrated submount alignment for optoelectronics", Journal of Lightwave Technology, 23, 504(2005)

[4] B. Li, H. Xie, B. Xu, R. Geer, J. Castracane, "Investigation of strain in microstructures by a novel moiré method", Journal of Microelectromechanical Systems, 11,829(2002)

[5] Wen-Hsien Chuang Luger, T. Fettig, R.K. Ghodssi, R. "Mechanical property characterization of LPCVD silicon nitride thin films at cryogenic temperatures", Journal of Microelectromechanical Systems, 13, 870 (2004) 